\title{
Instrumentelle Ausrüstung und bildgebende Dokumentation für die arthroskopische Chirurgie an großen Gelenken
}

\author{
घ Martin Hofmeister, Volker Bühren
}

\section{Zusammenfassung}

Arthroskopische Operationen haben sich von rein diagnostischen Eingriffen zu reparativen und rekonstruktiven Eingriffen entwickelt. Grundlage dafür war die rasante Entwicklung arthroskopischer Instrumente und Techniken. Optik, Mechanik und Elektronik müssen optimal aufeinander abgestimmt werden. Die elektronische Bildgebung, Bildverarbeitung und die digitale Dokumentation realisieren weitere Teilbereiche der digitalen Patientenakte.

\section{Arthroscopic Instruments and Image Capturing Documentation in Arthroscopic Surgery of the Large Joints}

Arthroscopic surgery has developed from a purely diagnostic procedure to reparative and reconstructive procedures. The basis for this was the rapid development of arthroscopic instruments and techniques. Synchronisation between optical, mechanical, and electronic devices is important. Electronic imaging, image processing, and digital documentation contribute additional information to the digital patient chart.

\section{Einleitung}

Die arthroskopische Behandlung großer Gelenke hat in den letzten Jahrzehnten enorme Fortschritte gemacht. So ist z.B. die Schulterarthroskopie nicht mehr auf resezierende Verfahren von Knochen und Weichteilgewebe oder die Entfernung freier Gelenkkörper begrenzt. Verbesserte OP-Technik und arthroskopische Instrumente erlauben die Ausdehnung der Indikationen $\mathrm{zu}$ rein arthroskopischen Versorgungen z.B. der Rotatorenmanschettenrekonstruktion. Auf dem Gebiet der Optik sind in den letzten Jahren kaum neue Entwicklungen entstanden, die Fertigung konnte bei optimierter Produktion Qualitätsverbesserungen erreichen. Die mechanischen Komponenten der Arthroskopie erfuhren wesentliche Verbesserungen durch Modifikation der Arthroskopieschäfte und Verschlussmechanismen. Moderne Pumpensysteme sorgen für gute Sichtverhältnisse und ermöglichen so zahlreiche arthroskopische Eingriffe. Druckerhaltende Arbeitskanülen erlauben schnellere

Portalwechsel bzw. Arbeitsabläufe ohne Kollabieren der Gelenkkapsel. Entsprechend der allgemeinen Entwicklung in der Medizin sind die größten Veränderungen der arthroskopischen Chirurgie auf dem Gebiet der bildgebenden und verarbeitenden Hard- und Software entstanden. Die Verwendung hochauflösender digitaler Chipkameras ist für die Erzeugung elektronischer Bilder unverzichtbar. Die Weiterleitung und Verarbeitung der Bilder in Mikroprozessortechnik bedarf aufeinander abgestimmterKomponenten.Diedurchgehend digitale Weiterleitung ermöglicht eine optimale Abstimmung der Faktoren Lichtstärke, Tiefenschärfe, Vergrößerung, Kontrast und Auflösungsvermögen bis hin zur Darstellung auf einem Flachbildmonitor. Die rechnerbasierte Aquisition und Intergration von Befunden ist Grundlage medizinischer Dokumentation. Die Archivierung digitaler Bilder kann auf lokalen Rechnern, CD-ROM, USB-Stick oder in Netzwerken. erfolgen.

\section{Arthroskop}

Für die Standardarthroskopie werden $4 \mathrm{~mm}$ Hopkins-Optiken mit einem $30^{\circ}$ -
Öffnungswinkel angewandt. Zur Beantwortung spezieller Fragen kann auch eine $70^{\circ}$-Optik eingesetzt werden. Arthroskope vertragen auch heutzutage keine Biegebelastung. Diese kann zu einer sichelförmigen Schwärzung des Sichtfeldrandes führen. Bricht das $\mathrm{Ar}$ throskop, wird es unbrauchbar. Es sollte nie ohne schützende Trokarhülse verwendet werden. Für die Arthroskopie der großen Gelenke von Schulter, Knie und Sprunggelenk werden standardmäßig $5 \mathrm{~mm}$-Rundarthroskopieschäfte verwendet. Am Ellenbogengelenk kommen sowohl $5 \mathrm{~mm}$ - als auch 2,8 mm-Schäfte zur

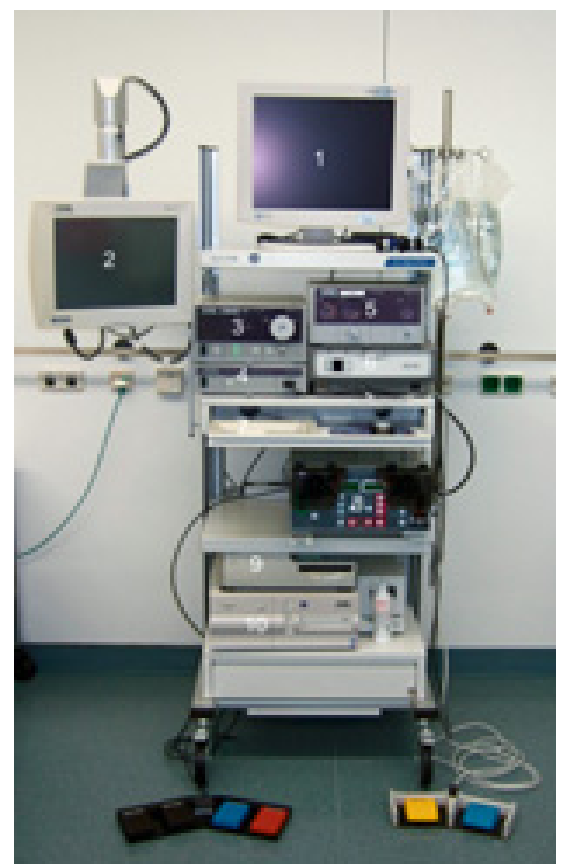

Abb. 1 Arthroskopie-Wagen Unfallklinik Murnau:

1 TFT-Flachbildschirm, 2 links mit TouchScreen zur Bedienung der Software, 3 XenonKaltlichtquelle, 4 Kamerakontrolleinheit, $5 \mathrm{CO}^{2}$ Arthroflator, 6 Arthrocare, 7 Tastatur und Maus für Dateneingabe oder Beschriftung, 8 fms ${ }^{\circledR}$ duo Pumpen- und Shaversystem , 9 Videoprinter, 10 Rechner für Bildverarbeitung, 11 Tricam-Kamera-Kopf, 12 Spülbeutel (3 Liter), 13 Fußschalter für Pumpensystem und Arthrocare. 
Anwendung. Am Handgelenk wird nur das Nadelarthroskop mit 2,8 mm eingesetzt (Abb.2) [1, 2]. Auf Wunsch können High-flow-Arthroskopschäfte mit erhöhter Durchflussrate der Spülflüssigkeit geliefert werden. Die zahlreichen Anbieter der Industrie haben verschiedenste anwenderfreundliche Schnellverschlussmechanismen entwickelt. Die Koppelung zwischen Optik und Schaft ist in jeder denkbaren Position möglich und erleichtert den Wechsel zwischen Arthroskop und Instrumentenzugang. Drehbare Anschlüsse für die Flüssigkeitszuleitung erleichtern durch vermehrte Bewegungsfreiheit die arthroskopische Operation. Zum Wechsel der verschiedenen Optiken bei einer Operation benötigt man einen Wechseladapter (Abb. 2).

\section{Spülpumpe}

Zahlreiche arthroskopische Eingriffe sind nur möglich, wenn einwandfreie Sichtverhältnisse durch kontinuierliche Perfusion des Gelenkes mit Ringer-Laktat erreicht werden. Dabei ist ein intraartikulärer Druck von $50 \mathrm{~mm} \mathrm{Hg}$ ausreichend zur Distension der Gelenkkapsel sowie zur Kompression kleiner synovialer Gefäße. Die gängigen Pumpensysteme sind mit einem Drucksensor ausgestattet und stellen bei Erreichen des voreingestellten Maximalwertes den Zufluss ab. Auf Wunsch kann bei vermehrter Blutungsneigung kurzfristig eine Erhöhung des intraartikulären Druckes veranlasst werden, dabei muss der Druck nicht über $70 \mathrm{~mm} \mathrm{Hg}$ angehoben werden. Als ernsthafte Komplikation ist jedoch immer die Entwicklung eines Kompartmentsyndromes zu bedenken, ggf. ist die Arthroskopie umgehend abzubrechen [2]. Die minimale Durchflussrate bei Spülfunktion sollte nicht unter 3 Liter pro Minute liegen. High-flow-Arthroskopieschäfte mit orthograder Spülrichtung sind in dieser Situation recht hilfreich. Bei Pumpensystemen, in denen Spülung und Absaugung kombiniert sind, regelt ein integrierter Rechner die Verschlussmechanismen. Nur hierdurch ist es möglich, bei konstantem Druck eine erhöhte Durchflussrate zu erreichen. Arbeitsintensive Verfahren werden vereinfacht, indem die intergrierte Saugpumpe die Shaver- oder Kanülenabsaugung automatisch anwählt. Mittels der eingangsund ausgangsseitigen Regeltechnik werden Druck und Durchfluss genau geregelt. Bei Rotation des Shavers schaltet das $\mathrm{duO}^{\circledR}+$ System automatisch auf Absaugung durch den Shaver und klemmt die Kanülensaugung ab [8].

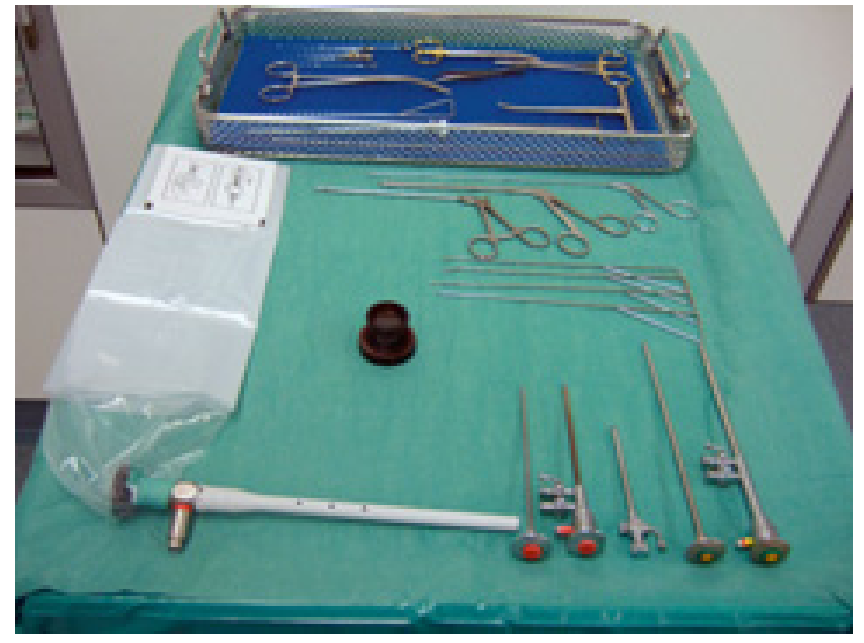

Abb. 2: Basisinstrumentarium Arthroskopie: 2,8 mm- und $5 \mathrm{~mm}$ Arthroskopieschäfte, Arthroskop mit Schutzhülse, Wechseladapter, spitze und stumpfe Taststäbe, Fasszange, PE-Zange, Rongeur.

Bei umgekehrter Stromrichtung mit Saugung am Arthroskop werden Blut und Gewebsfetzen in das Gesichtsfeld gesaugt und die Sicht wird damit verschlechtert. Arbeitstrokare mit Lippenventilmechanismus ermöglichen die Aufrechterhaltung des intraartikulären Druckes auch bei häufigem Wechsel arthroskopischer Instrumente. Die $\mathrm{Ar}$ throskopie unter $\mathrm{CO}_{2}$-Gasfüllung wird nur noch bei rein diagnostischen Eingriffen angewendet.

\section{Instrumente}

$\mathrm{Zu}$ den Standardinstrumenten der arthroskopischen Chirurgie gehören spitze und stumpfe Taststäbe evtl. auch mit Millimeterangabe, Rongeure, Fasszange und ggf. ein Wechselstab (WissingerStab) zur Anlage neuer Portale (Abb. 2).

Zusatzinstrumente müssen dem jeweiligen Einsatz entsprechend ausgewählt werden. Je nach individuellem Gewebetyp und vorhandenen degenerativen Veränderungen sind bei biologischem Gewebe unterschiedliche Widerstände vorzufinden. Das reicht von degenerativen zerfaserten Anteilen über Kalkeinlagerungen und Vernarbungen bis zu partiellen Verknöcherungen. Aus diesem Grunde stehen zahlreiche arthroskopische Instrumente mit unterschiedlichen Öffnungsrichtungen, Maulformen und Abwinkelungen der minimalinvasiven Chirurgie zur Verfügung (Fasszangen, Stanzen, Scheren, Biopsiezangen, Messer, Küretten, Raspeln). Darüber hinaus finden zahlreiche Spezialinstrumente, die hier im Einzelnen nicht dargestellt werden können, regelmäßigen Einsatz (z. B. Knochenanker, Sehnenfasszangen, Zielinstrumentarien, Meniskusnähte etc.)

\section{Shaver}

Motorgetriebene Shaver-Systeme zahlreicher Anbieter finden insbesondere zur Weichteilbehandlung häufig Anwendung. Der Sog zieht Gewebe durch ein Fenster in ein Saugrohr, die rotierende oder oszillierende Klinge trennt es ab. Wichtigste Voraussetzung für die Wirksamkeit der motorgetriebenen Instrumente ist ein ausreichendes Flüssigkeitsangebot. Zur Glättung von Knochenvorsprüngen kommen hochfrequent rotierende Fräsen (8000-12000 Umdrehungen) als Einmalartikel zum Einsatz. Die Resterilisierung ist aus hygienischen Gründen leider nicht zugelassen (Abb. 3 und 4) $[6,8]$.

\section{Elektrochirurgie}

Arthroskopische Elektroden werden seit Jahren zur Blutstillung und zum Schneiden bei arthroskopischen Eingriffen, wie dem lateralen Release und der subakromialen Dekompression, verwendet. Radiochirurgische Systeme gehen über die Ablation von Gewebe hinaus und umfassen auch Operationen an Gelenkknorpel, Menisken, Gelenkkapsel und Bandstrukturen. Monopolare und bipolare Ablationssonden stehen in zahlreichen Größen, Abwinkelungen und Formen der Elektroden tragenden Spitze zur Verfügung, sodass jeder Gelenkabschnitt leicht zu erreichen ist. Bei der bipolaren radiochirurgischen Technik wird zwischen den eng benachbarten Elektroden ein lokales Plasmafeld an der Sondenspitze aufgebaut. Dies bewirkt die Desintegration von Molekülen im behandelten Gewebe. Im leitenden Medium der Spülflüssigkeit werden organische molekulare Verbindungen innerhalb des Gewebes durch ionisierende Teilchen 


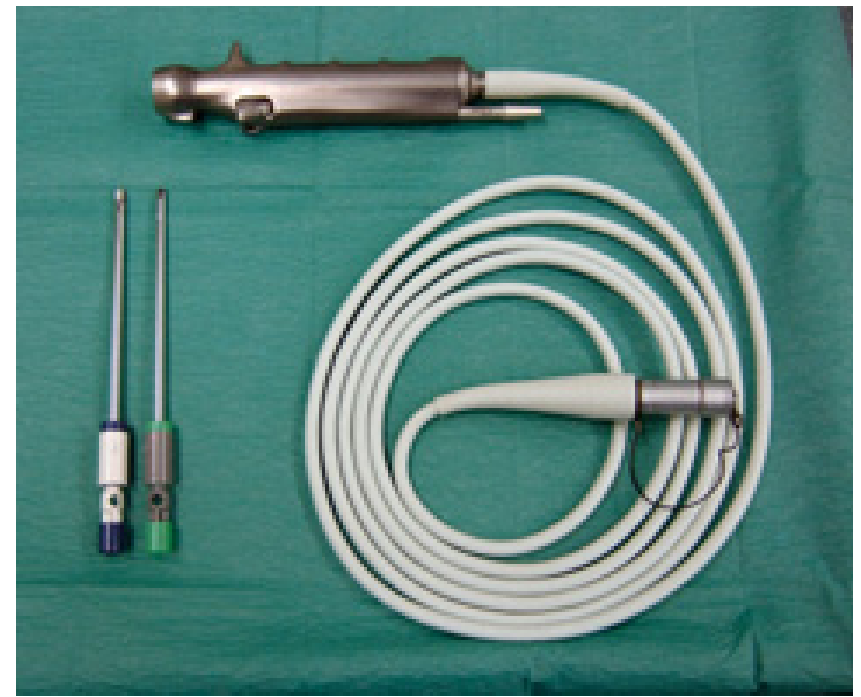

Abb. 3 Fms ${ }^{\circledR}$ duoShaver-Handstück mit Shaver-Klinge und Akromizer.

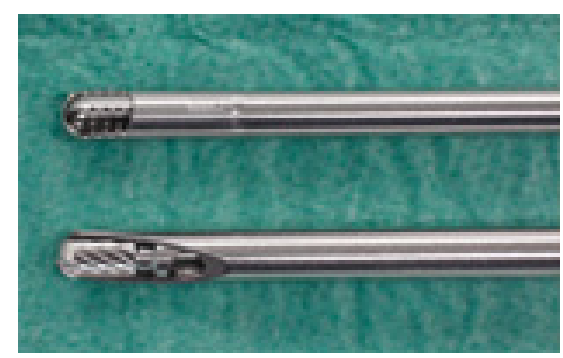

Abb. 4 Detailansicht Shaver- und AkromizerSpitzen.

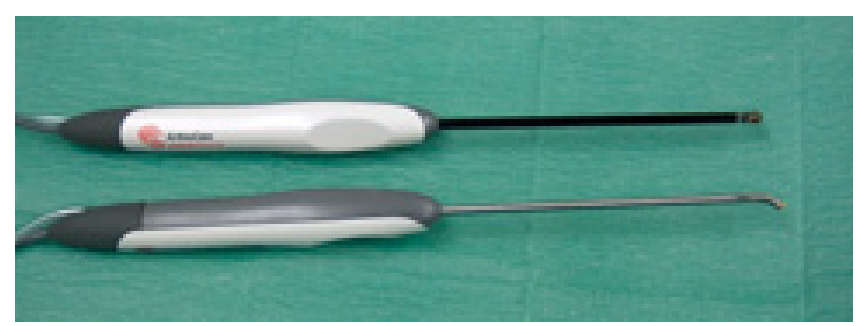

Abb. 5 ArthrocareHandstücke Turbovac und Tristar 50. aufgebrochen. Elementare Moleküle und Gase niedrigen Molekülgewichts werden mit der Spülflüssigkeit abtransportiert [4]. Bei diesem Vorgang entstehen Temperaturen von $70^{\circ} \mathrm{C}$. Die Spülflüssigkeit darf zur Vermeidung von Verbrühungen nicht über die Haut abfließen, weshalb die Sonden mit einem Absaugkanal ausgestattet sind. Durch den räumlich begrenzten Stromfluss werden zahlreiche Nachteile und Gefahren monopolarer oder Lasersysteme vermieden. Bislang ist die Eindringtiefe des Plasmafeldes und damit die Auswirkung auf tiefer liegende Knorpelschichten bei der Knorpelglättung nicht eindeutig geklärt. Aus diesem Grunde werden Radiochirurgiesysteme von manchen Autoren für diese Indikation abgelehnt (Abb.5 und 6).

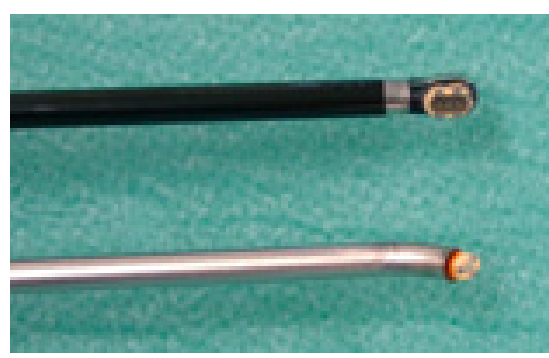

Abb. 6 Detailansicht Arthrocare-Spitzen z. B. Turbovac und Tristar 50.

\section{Kaltlichtquelle und Lichtkabel}

Bei den Kaltlichtquellen stehen Halogenund Xenonlampen zur Verfügung. Die stufenlos manuelle oder über die Kamera verstellbare Lichtintensität erlaubt eine individuelle Anpassung. Die Farbtemperatur beträgt 3400 bis 6000 Kelvin. Die Tageslichtqualität des Xenonlichtes kommt den normalen Sehgewohnheiten des Menschen entgegen. Fiberglaslichtkabel stehen in den Längen von 230 bis $380 \mathrm{~cm}$ zur Verfügung. Durch die hohe Lichtkonzentration am Ende des Lichtkabels entsteht im Brennpunkt Wärme. Das Ende des Lichtkabels darf nicht auf der Haut des Patienten oder der OP-Abdeckung abgelegt werden. Es können Verbrennungen der Patientenhaut oder ein Inbrandsetzen der Abdeckung hervorgerufen werden. Mit spezieller Flüssigkeit gefüllte Fluid-Lichtkabel sind steifer als Fiberglas-Lichtkabel. Sie dürfen nicht so stark gekrümmt oder geknickt werden. Das von ihnen übertragene Licht ist intensiver und deshalb vorzugsweise für endoskopische Photographie, Film und Fernsehaufnahmen geeignet [5].

\section{Digitale Dokumentation Bildgebender Diagnostik und Therapie:}

\section{Kamera}

Im Chipkamerakopf nimmt über ein speziell beschichtetes Farbprisma eine Trennung in die drei Primärfarben vor. Jeder einzelne von drei CCD-Sensoren erhält somit bewusst nur eine der Farbinformationen, bei jeweils maximaler Zeilendarstellung. Durch die separate Verarbeitung der Primär-Farbinformationen Rot-Grün-Blau, werden nur mit diesem Verfahren sämtliche Farben optimal erkannt. Die Signalverarbeitung erfolgt im digitalen Bildprozessor. Bei der Aufbereitung übernimmt der Bildprozessor die automatische Bildoptimierung bei ständig wechselnden Beleuchtungs- und Farbverhältnissen [5]. Technisch sind Auflösungen von 3 x 800000 bis 3 x 1,2 Mega Pixel möglich. Große Hoffnung zur Optimierung der Bildqualität setzen Anwender und Industrie in die Entwicklung der Real-HDTV-Kameras. Völlig neue Bildgebungsmöglichkeiten werden auch von der „Chip on the Stick“-Technik erwartet. Hierbei wird ein Sensorchip direkt am Ende des Schaftes platziert. Ob sich hierdurch „neue Einblicke“ in die Gelenke ergeben, muss abgewartet werden.

\section{Monitor}

Mit großflächigen 19“ TFT-Flatscreens erreicht man eine optimale Darstellung der digitalen Bilder in bester Auflösung und Kontrast. Erst seit der Reduktion der Reaktionszeiten auf $5 \mathrm{~ms}$ sind bewegte Bilder in brauchbarer Qualität zu erhalten. Touch-Screen-Oberflächen lassen die komfortable Bedienung der Software, Überwachung und Kontrolle direkt aus dem sterilen Bereich oder über einen Springer zu.

Die direkte Darstellung des arthroskopischen Bildes ist nach wie vor die unbestechlichste Form der Dokumentation eines operativen Eingriffs. Hierzu sind teure und ineffiziente Farbvideo-Drucker noch weitverbreitet. Die Dokumentation 


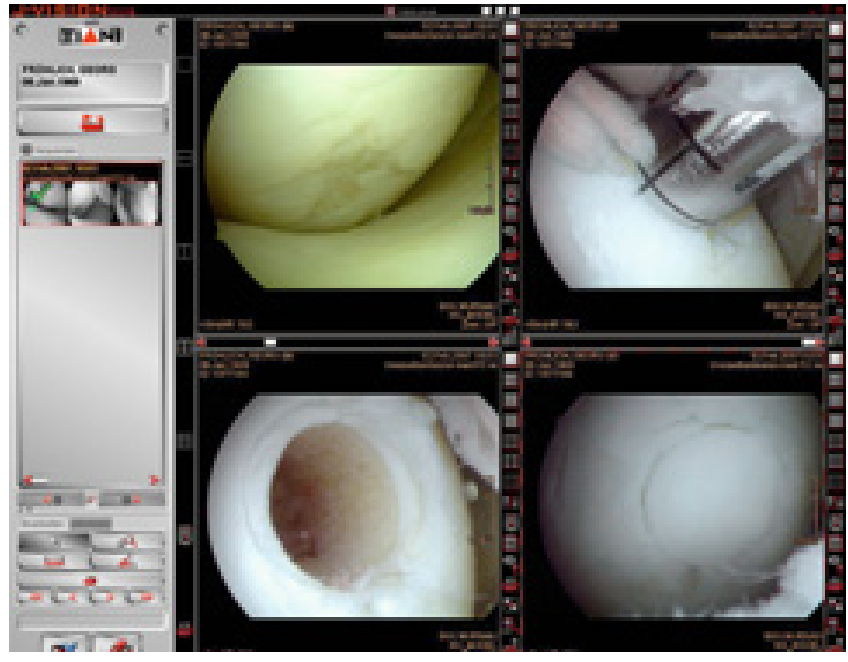

Abb. 7 Arthroskopische Bildgebung im J-Vision-TIANI. OATS-Plastik medialer Femurkondylus bei umschriebenem Knorpelschaden.

digitaler Daten mit minimalem Erfassungsaufwand setzt eine leistungsfähige Krankenhaus-IT-Infrastruktur voraus. Die Speicherung im Netzwerk, auf DVD und CD-ROM sorgt für eine ergiebige Datenarchivierung und ermöglicht durch die Mobilität der Daten deren Zugang an nahezu jeder beliebigen Stelle. Die Verwendung z.B. im PACS ermöglicht die Demonstration von Arthroskopiebefunden in einer Röntgen-Abteilungsbesprechung (Abb. 7). Mehrere Startkonfigurationen können individuell vordefiniert und nach Bedarf aufgerufen werden. So können verschiedene Abteilungen ohne das manuelle Einstellen vor jeder OP Geräte und Software abteilungsübergreifend verwenden. Bei wissenschaftlichen Publikationen kann auf die gespeicherten Bilder zugegriffen werden.

\section{Arthroskopie Bilddokumentation Unfallklinik Murnau}

Das zurzeit in der Unfallklinik verwendete System AIDA compact II (Fa. Karl Storz, Tuttlingen ) zeichnet Standbilder und Videosequenzen von Befunden direkt aus dem sterilen Bereich auf. Nach Einschalten der Arthroskopieeinheit wird automatisch eine Liste des Tages-OP-Programms angezeigt. Nach Auswahl des Patienten werden die Pflichtdaten Nachname, Vorname, Geburtsdatum, Diagnose und geplante OP aus der OP-Anmeldung in die Software des bildverarbei- tenden Systems übernommen. Die OP kann beginnen. Per Fingertip auf dem Touchscreen, Fußschalter oder Kameraknopfdruck können diese Aufnahmen ausgelöst werden. Live-Darstellungen der Kamerabilder am Touchscreen ermöglichen eine sofortige Kontrolle und Selektion der aufgezeichneten Daten. Im Bearbeitungsbildschirm können die zwischengespeicherten Daten vor der endgültigen Archivierung nochmals angesehen werden. Nicht benötigte Daten werden einfach gelöscht. Die einzelnen Bilder und Videodateien können bei Bedarf umbenannt und mit aussagefähigen Bezeichnungen versehen werden, wobei eine voreinstellbare Auswahlliste mit Schlüsselwörtern die Dateneingabe erleichtert und beschleunigt. Außerdem steht ein Kommentarfeld zur Verfügung, in das Anmerkungen $\mathrm{zu}$ interessanten Details des Eingriffs eingetragen werden können. Noch während des Betrachtens der Video- und Bilddateien kann per Spracheingabe der OP-Bericht akustisch aufgezeichnet werden. Die technischen Probleme einer Spracherkennung der Audiodateien und die Umwandlung in Textdateien sind im Hause noch nicht befriedigend umgesetzt. Nach Abschluss einer Behandlung sichert AIDA compact II selbstständig die Daten über einen drahtlosen W-LAN-Anschluss in ein vorbestimmtes Archiv. Alternativ kann auch die Speicherung auf DVD, CD-ROM oder einen USB-Stick erfolgen. Zusätzlich kann ein Standardbericht mit Bild und Text der Operation erstellt und bei Bedarf als Übersichtsinformation ausgedruckt werden. Sofern keine Netzwerkarchivierung im Klinik-InformationsSystem (KIS) vorgesehen ist, kann eine effiziente Datenarchivierung durch Speicherung mehrerer Behandlungen auf einer CD-ROM oder DVD garantiert werden. Sämtliche Geräte sind für den Einsatz im OP nach EN 60601-1 zugelassen [5]. Die Verwendung von Videoprinter oder Videorecorder kann entfallen.

\section{Fazit}

Die Arthroskopie großer Gelenke hat sich aufgrund der technischen Entwicklungen von Optik, Bildgebung und arthroskopischer Instrumente $\mathrm{zu}$ einer hoch spezialisierten Gelenkchirurgie entwickelt. Im Zeitalter digitaler Datenverarbeitung ist mit der Bildgebung, Bildverarbeitung und Dokumentation ein weiterer Schritt in Richtung der elektronischen Patientenakte vollzogen.

\section{Literatur}

1 Hempfling $\mathrm{H}$. Farbatlas der Arthroskopie großer Gelenke. 2. Auflage Gustav Fischer, Stuttgart, 1995

2 Imhof A, Ticker B, Fu F. An Atlas of Shoulder Arthroskopie. Martin Dunitz, London. 2003

3 Kohn D. Diagnostische und operative $\mathrm{Ar}$ throskopie großer Gelenke. Thieme Stuttgart, 1997

4 Wienecke H, Lobenhofer P. Grundlagen radiochirurgischer Systeme und ihre Anwendung in der Arthroskopie Arthroskopie 2003; 15: 110-120

5 Fachinformation der Firma Karl Storz GmbH und Co. KG Tuttlingen 2006

6 Fachinformation der Firma Arthrex $\mathrm{GmbH}$ Karlsfeld/München

7 Fachinformation der Firma Arthrocare Sunnyvale, Kalifornien, USA

8 Fachinformation fmsgroup International Geneva Schweiz

\section{Dr. med. Martin Hofmeister Oberarzt}

Prof. Dr. med. Volker Bühren

Ärztlicher Direktor

Berufsgenossenschaftliche

Unfallklinik Murnau

Professor-Küntscher-Straße 8

82418 Murnau am Staffelsee 